\title{
Kondo Force in Shuttling Devices: Dynamical Probe for a Kondo Cloud
}

\author{
M.N. Kiselev, ${ }^{1}$ K.A. Kikoin, ${ }^{2}$ L.Y. Gorelik, ${ }^{3}$ and R.I. Shekhter ${ }^{4}$ \\ ${ }^{1}$ The Abdus Salam International Centre for Theoretical Physics, Strada Costiera 11, I-34151 Trieste, Italy \\ ${ }^{2}$ School of Physics and Astronomy, Tel-Aviv University, Tel-Aviv 69978, Israel \\ ${ }^{3}$ Chalmers University of Technology, Department of Applied Physics, SE-412 96 Göteborg, Sweden \\ ${ }^{4}$ University of Gothenburg, Department of Physics, SE-412 96 Göteborg, Sweden
}

(Dated: June 19, 2021)

\begin{abstract}
We consider electromechanical properties of a single-electronic device consisting of movable quantum dot attached to a vibrating cantilever, forming a tunnel contact with a non-movable source electrode. We show that the resonance Kondo tunneling of electrons amplify exponentially the strength of nanoelectromechanical (NEM) coupling in such device and makes the latter to be insensitive to mesoscopic fluctuations of electronic levels in a nano-dot. It is also shown that the study of KondoNEM phenomenon provides an additional (as compared with a standard conductance measurements in a non-mechanical device) information on retardation effects in formation of many-particle cloud accompanied the Kondo tunneling. A possibility for superhigh tunability of mechanical dissipation as well as supersensitive detection of mechanical displacement is demonstrated.
\end{abstract}

PACS numbers: 73.23.-b, 72.10.Fk, 73.23.Hk, 85.85.+j

Recent progress in fabrication of nanoelectromechanical systems (NEMS) based on suspended carbon nanotubes [1] as well as on suspended $\mathrm{Si}[2$ and $\mathrm{SiN}[3]$ nanowires vibrating at radio frequencies $(\mathrm{RF})$ resulted in rapidly growing amount of theoretical works [4-8] addressing the issues of interplay between spin/charge transport and nano-mechanics 9. The observation of Coulomb Blockade 2] in NEMS opened a possibility to consider the influence of strongly correlated and resonance effects on a behaviour of nano-oscillators.

Usually, NEM regime implies strong coupling between the electronic and mechanical degrees of freedom. The coupling is provided by two main mechanisms. Motion of the movable dot (shuttle) between two metallic banks results in the time dependent tunneling amplitudes. On the other hand, the electron charge transport between the banks in presence of magnetic field results in appearance of a Lorentz/Laplace force acting on the shuttle, which should also be taken into account 4, 5. The aggregate dynamics of a shuttle is that of a periodic oscillator with decrement or increment and an electron tunneling (cotunneling) parametrically dependent on this slow classical motion. In some sense the problem may be treated as a tunneling through anharmonic vibronic system. In many cases, e.g. in shuttling devices including bending carbon nanotubes [5], the vibronic language completely describes the physical situation.

NEM coupling like other nanometer length scale phenomena is strongly affected by mesoscopic fluctuations. Spatial quantization of electronic motion in a quantum dot makes electro-mechanical transduction to be sample sensitive phenomenon [2. An exception to this rule is electromechanical coupling due to the many-body Kondo tunneling. Indeed in this case the charge transfer is controlled by the singularity of the tunneling density of states at the energy pinned to the Fermi level of the injec- tor and thus protected against mesoscopic fluctuations. This fact in combination with another generic feature of Kondo phenomenon - its super-sensitivity to a strength of the tunneling coupling (and therefore its super-sensitivity to the mechanical displacement of a quantum dot) makes Kondo NEM coupling to be a phenomenon promising for practical applications.

An example of such device is schematically shown in Fig. 1. A nanoisland is mounted on the metallic cantilever, which may vibrate under an external force. The contact between the source and drain electrodes is a combination of time-dependent tunneling bridge between the source $S$ and the island and a metallic bridge formed by vibrating cantilever connecting the island with the drain $D$ (see Ref. 2 for experimental realization).

We consider the configuration where a cantilever is displaces in $y$ direction, $\vec{u}=(0, y, 0)$ in a magnetic field $\vec{B}=$ $(0,0, B)$. In this case the Laplace force $\vec{F}$ acts on the can-

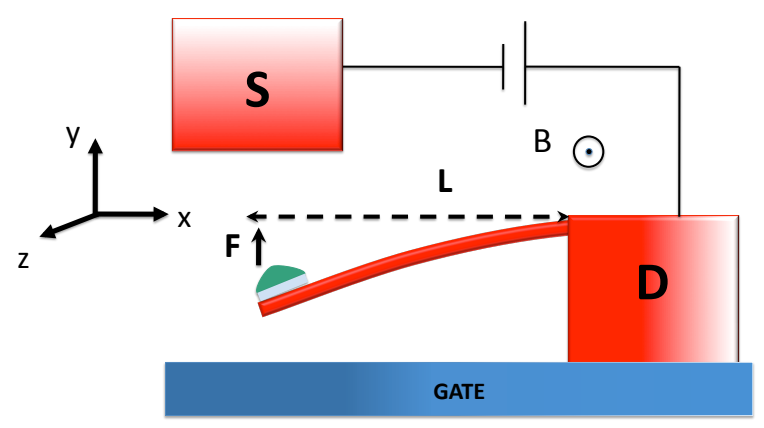

FIG. 1. (Color online) Shuttle with a cantilever. The nanoisland (green) is separated from the wire (drain) by a tunnel barrier (light blue) of constant width. The width of the barrier to the source changes during the cycle. Shuttle oscillations are stimulated by initial conditions, e.g. bending the cantilever by the gate voltage at time $t=0$. 
tilever in the same direction $y, \vec{F}_{L}=L \cdot \vec{I} \times \vec{B}=(0, F, 0)$. Here $L$ is the length of the cantilever. Besides, small electromotive force $\vec{F}_{\text {emf }}=(f, 0,0)$ acts on the electrons in the cantilever. In the limit of strong Coulomb blockade in the nanoisland, the Kondo screening accompanies the tunneling 'source-island-cantilever', and a unique possibility arises to study the contribution of purely quantum many-particle Kondo effect on the classical oscillation of a shuttle (cantilever + island). The study of a "Kondo force" in shuttling is the main subject of this paper.

We study two coupled subsystems: a tunneling contact 'source-moving island-moving cantilever' treated as a purely quantum system in a framework of the Anderson - Kondo model, and a macroscopic wire with attached island oscillating under external constraining force. We work in the Kondo limit, where the nanoisland is represented by its spin $\vec{S}$, so that internal degrees of freedom are the spin-flip processes. The source-drain transport is a combination of quantum tunneling 'source - island - cantilever' and ohmic transport 'island-drain'. In this "time-dependent Schrieffer - Wolff" limit (see below) the Hamiltonian of quantum subsystem is

$$
\begin{gathered}
H=H_{\text {lead }}+H_{\text {ex }}+\delta H \\
H_{\text {lead }}=\sum_{\alpha=l, r} \sum_{k \sigma} \xi_{k} c_{\alpha k \sigma}^{\dagger} c_{\alpha k \sigma}, \quad H_{\mathrm{ex}}=\sum_{\alpha \alpha^{\prime}} J_{\alpha \alpha^{\prime}} \vec{s}_{\alpha \alpha^{\prime}} \cdot \vec{S} \\
\delta H=\frac{e V_{\text {bias }}}{2}\left(N_{l}-N_{r}\right)
\end{gathered}
$$

Here the indices $l, r$ stand for the electronic states in the source and cantilever, respectively, $\xi_{k}=\varepsilon_{k}-\mu$ are the excitation energies of lead electrons, $N_{\alpha}=\sum_{k \sigma} n_{\alpha k \sigma}$ are the corresponding electron density operators, $\vec{s}_{\alpha \alpha^{\prime}}=$ $\frac{1}{2} \sum_{k k^{\prime}} c_{\alpha k \sigma}^{\dagger} \vec{\tau}_{\sigma \sigma^{\prime}} c_{\alpha^{\prime} k^{\prime} \sigma^{\prime}}, \vec{S}=\frac{1}{2} d_{m}^{\dagger} \vec{\sigma}_{m m^{\prime}} d_{m^{\prime}}$ are the spin operators for the electrons in the leads and in the nanoisland, respectively, $\vec{\tau}$ and $\vec{\sigma}$ are the vectors of Pauli matrices acting on the states in the leads and dot. At small bias voltage $V_{\text {bias }}$ the source and the cantilever are supposed to be in the adiabatically stationary state of thermal equilibrium. The parameters are $J_{\alpha \alpha^{\prime}}=4 v_{\alpha}^{*} v_{\alpha^{\prime}} / E_{c}$, where $v_{\alpha}$ is the tunneling amplitude between the nanoisland and the metallic lead $\alpha, E_{c}$ is the Coulomb blockade energy. The exchange couplings $J_{l l}$ and $J_{l r}$ are timedependent due to the dependence of the tunneling amplitude between the source and the moving nanoisland on the island position $v_{l}=v_{l}[\vec{u}(t)]$. The time dependence of this amplitude is a set of pulses corresponding to electron injection from the metallic reservoir to the shuttle periodically approaching the bank $S$. [10] We confine our treatment with the simplest case of $S=1 / 2$ (odd occupation of a nanoisland in the neutral state) and the single channel tunneling between the nanoisland and the leads.

The oscillations of cantilever with attached nanoisland are determined by the classical Newton equations

$$
\ddot{\vec{u}}+\frac{\omega_{0}}{Q_{0}} \dot{\vec{u}}+\omega_{0}^{2} \vec{u}=\frac{1}{m} \vec{F} .
$$

where $\omega_{0}=\sqrt{k / m}$ is the oscillator frequency of free cantilever, $Q_{0}$ is a quality factor of NEM device.

Our aim is to study the spin and charge transport by means of a shuttle oscillating in accordance with Eq. (2) in presence of many-particle Kondo screening described by the Hamiltonian (1). The coupling between the classical and quantum subsystem is realized via the parameters $J_{l l}(\vec{u}), J_{l r}(\vec{u}), \vec{F}(\vec{u})$, where the time dependence $\vec{u}(t)$ should be calculated self-consistently. Meanwhile, $J_{r r}$ does not depend on displacement $\vec{u}$ (see Fig. 1).

The cotunneling Hamiltonian may be rationalized by means of the Glazman-Raikh rotation, which in our situation is time dependent:

$$
\left(\begin{array}{c}
c_{l k \sigma} \\
c_{r k \sigma}
\end{array}\right)=\left(\begin{array}{cc}
\cos \vartheta_{t} & -\sin \vartheta_{t} \\
\sin \vartheta_{t} & \cos \vartheta_{t}
\end{array}\right)\left(\begin{array}{l}
\psi_{1 k \sigma} \\
\psi_{2 k \sigma}
\end{array}\right) \equiv U_{t}\left(\begin{array}{l}
\psi_{1 k \sigma} \\
\psi_{2 k \sigma}
\end{array}\right)
$$

with $\tan \vartheta_{t}=\left|v_{r} / v_{l}(t)\right|$. Use of this transformation for diagonalization of the Schrödinger operator $\mathcal{L}=$ $-i \hbar d / d t+H(t)$ results in generation of additional term $H_{B}$ proportional to $-i \hbar U_{t}^{-1} \partial_{t} U$ in the transformed Hamiltonian (see, e.g., [14]).

$$
\begin{gathered}
H^{\prime}=H_{\text {lead }}+H_{\mathrm{B}}+H_{\mathrm{ex}}+\delta H \\
H_{\text {lead }}=\sum_{a=1,2} \sum_{k \sigma} \xi_{k} \psi_{a k \sigma}^{\dagger} \psi_{a k \sigma} \\
H_{\mathrm{B}}=i \hbar \frac{d \vartheta_{t}}{d t} \sum_{k \sigma}\left(\psi_{1 k \sigma}^{\dagger} \psi_{2 k \sigma}-\psi_{2 k \sigma}^{\dagger} \psi_{1 k \sigma}\right) \\
H_{\mathrm{ex}}=\frac{J}{4} \sum_{k k^{\prime}, \sigma \sigma^{\prime}, m, m^{\prime}} \psi_{1 k \sigma}^{\dagger} \vec{\tau}_{\sigma \sigma^{\prime}} \psi_{1 k^{\prime} \sigma^{\prime}} d_{m}^{\dagger} \vec{\sigma}_{m m^{\prime}} d_{m^{\prime}},(5) \\
\delta H=\frac{e V_{\mathrm{bias}}}{2}\left[\left(N_{2}-N_{1}\right) \cos 2 \vartheta_{t}+\sum_{k \sigma}\left(\psi_{1 k \sigma}^{\dagger} \psi_{2 k \sigma}+h . c .\right) \sin 2 \vartheta_{t}\right]
\end{gathered}
$$
The term $H_{B}$ may be treated as an additional gauge potential in the lead Hamiltonian describing a Berry-like phase 14 generated by shuttle motion [13]. Only the even partial wave $\psi_{1}$ survives in the cotunneling term $H_{\text {ex }}$ with the time-dependent effective indirect exchange coupling $J(t)=J_{l l}(t)+J_{r r}$ (see, e.g., [15]). This time dependence may be parametrized [9] in assumption that the source-island tunneling amplitude is an exponential function of a distance $y$ between the source and the moving nanoisland, while the tunneling nanoislandcantilever is constant: $v_{r}=v_{0}, \quad v_{l}=v_{1} \exp [y(t) / \lambda]$. The spatial coordinates are counted off the equilibrium position of cantilever, so that $v_{1}$ is exponentially small, $v_{1} / v_{0} \sim \exp \left(-y_{0} / \lambda\right)$. Here $\lambda$ is the confinement radius (tunnel length) of the electron wave function within the island, $y_{0}$ is the distance between the source and the island at equilibrium.

We suppose that the shuttling mode is slow enough and the electron transport is adiabatic, i.e., the exchange couplings $J_{l l}, J_{l r}$ depend parametrically on time via the displacement coordinates $\vec{u}(t)$. Then to find the tunneling 
current one may trace the time dependence of local occupations of the left and right banks (source and nanoisland) near the point of tunneling contact. The current operator is

$$
\hat{\mathcal{I}}=\frac{e}{2} \frac{d}{d t}\left(\hat{N}_{r}-\hat{N}_{l}\right)
$$

where in the case of immovable nanoisland only the even mode 1 contributes to the current. In our case both modes 1,2 are involved in the tunneling transport due to the term $H_{\mathrm{B}}$ in Eq. (4). After the Glazman-Raikh rotation the current operator transforms into

$$
\hat{\mathcal{I}}=\frac{d}{d t} \hat{\mathcal{Q}}_{t}+\frac{d}{d t} \hat{q}_{t}
$$

where

$$
\begin{gathered}
\hat{\mathcal{Q}}_{t}=\frac{e}{2} \cos 2 \vartheta_{t}\left(\hat{N}_{1}-\hat{N}_{2}\right) \\
\hat{q}_{t}=-\frac{e}{2} \sin 2 \vartheta_{t} \sum_{k \sigma}\left(\psi_{1 k \sigma}^{\dagger} \psi_{2 k \sigma}+\psi_{2 k \sigma}^{\dagger} \psi_{1 k \sigma}\right)
\end{gathered}
$$

Here the operator $\hat{\mathcal{Q}}_{t}$ controls the time-dependent electron occupation in the source lead, and the operator $\hat{q}_{t}$ is responsible for all tunneling and cotunneling processes including admixture of odd components $\psi_{2 k \sigma}$ to the tunneling charge transport induced by the gauge field $H_{B} \cdot 14,16$,

The time-dependent Glazman-Raikh angle defined by (9) results in adiabatic time dependence of the BreitWigner factor

$$
\sin ^{2} 2 \vartheta_{t}=\frac{4 \Gamma_{l} \Gamma_{r}}{\left(\Gamma_{l}+\Gamma_{r}\right)^{2}}=\frac{1}{\cosh ^{2} \frac{\left[y(t)-y_{0}\right]}{\lambda}}
$$

Using the Friedel - Langreth sum rule [17, one may write

$$
N_{1}-N_{2}=\frac{\delta_{t}}{\pi} .
$$

where $\delta_{t}=\delta_{\uparrow}+\delta_{\downarrow}$ is a total time dependent Friedel phase. At the unitary limit $\delta_{\uparrow, \downarrow}= \pm \pi / 2$.

We are interested in the Kondo effect contribution to the tunneling current. This contribution is characterized by the spin dependent scattering phase shift $\delta_{\sigma}(\varepsilon)$ in the source lead, which approaches the unitarity limit $\pi / 2$ at $T \rightarrow 0$ and $\varepsilon \rightarrow \varepsilon_{F}$. In the adiabatic limit $\hbar \omega_{0} \ll k_{B} T_{K}^{\min }$ under conditions $\left(k_{B} T, g \mu_{B} B,\left|e V_{\text {bias }}\right|\right) \ll k_{B} T_{K}^{\text {min }}$ the phenomenological Fermi liquid Hamiltonian $H_{\text {Noz }}$ may be used [18] (here $k_{B}$ is Boltzmann constant, $\mu_{B}$ is Bohr magneton and $g$ is Landé factor). In this Hamiltonian both scattering and interaction are scaled by the time-dependent Kondo temperature $T_{K}(t)$ taking minimal value $T_{K}^{\min }$ at maximal distance from the source.

In order to get full tunnel current in adiabatic approximation we (i) calculate a linear response with respect to both bias $e V_{\text {bias }} \ll k_{B} T_{K}$ and $\hbar d \vartheta_{t} / d t \leq$ $\hbar \omega_{0} \vartheta_{\max } \ll k_{B} T_{K}$, (ii) take into account cancellations arising due to emergent $S U(2)$ symmetry associated with channels [13, 20, (iii) perform averaging with the adiabatic Hamiltonian (4.5) at zero bias and zero temperature. The finite temperature and bias effects are accounted by Nozieres method. [18, [19] As a result, the tunnel current $\overline{\mathcal{I}}_{t}=\bar{I}_{0}(t)+\bar{I}_{\text {int }}(t)$ consists of two parts: the Friedel phase contribution

$$
\bar{I}_{0}(t)=\frac{e}{2 \pi} \cos 2 \vartheta_{t} \cdot \frac{d \delta_{t}}{d t}
$$

and the "ohmic" current 21]

$$
\bar{I}_{\mathrm{int}}(t)=\frac{e^{2}}{\hbar} V_{\mathrm{bias}} \frac{d}{d t}\left[\sin 2 \vartheta_{t} \int_{-\infty}^{t} d t^{\prime} \sin 2 \vartheta_{t^{\prime}} \Pi_{t-t^{\prime}}^{R}\right]
$$

where

$\Pi_{t}^{R}=-\frac{i}{2} \sum_{k \sigma} \sum_{\alpha \neq \gamma}\left[G_{\alpha k \sigma}^{R}(t) G_{\gamma k \sigma}^{K}(-t)+G_{\alpha k \sigma}^{K}(t) G_{\gamma k \sigma}^{A}(-t)\right]$

and $G_{\alpha=1,2}^{\Lambda}$ are bold retarded $(\Lambda=R)$, advanced $(\Lambda=A)$ and Keldysh $(\Lambda=K)$ Green's function [21.

Let us first rewrite the Friedel part of the tunneling current (11) via the parameters characterizing the Kondo tunneling in the low-temperature strong coupling limit at $T \ll T_{K}^{\min }$, where

$$
k_{B} T_{K}(t)=D_{0} \exp \left[-\frac{\pi E_{c}}{4\left(\Gamma_{l}+\Gamma_{r}\right)}\right],
$$

$D_{0}$ is the ultraviolet cut-off for the Kondo problem with a scale of the band energy in the source, $\Gamma_{\alpha}=\pi \rho_{0}\left|v_{\alpha}\right|^{2}$, $\rho_{0}$ is the density of electronic states at the Fermi level $\varepsilon_{F}$. In our adiabatic regime $T_{K}$ parametrically depends on time, following the time dependence of $\Gamma_{l}(t)$. The Hamiltonian $H_{\text {Noz }}$ [18, [19] establishes the relations between $\delta_{\sigma}, B$ and $T_{K}$ near the unitary limit, such as $\delta_{t}=2\left|e V_{\text {bias }}\right| /\left(k_{B} T_{K}(t)\right) \ll 1$. The magnetic field enters only into relative Friedel phase $\delta_{\uparrow}-\delta_{\downarrow}=\pi-$ $2 g \mu_{B} B /\left(k_{B} T_{K}(t)\right)$. We neglect the influence of magnetic field on $T_{K}$, since we work in the limit $g \mu_{B} B \ll T_{K}^{m i n}$. Alternatively, a non-uniform magnetic field negligible at the dot and gradually increasing along the cantilever could be assumed in the model.

In the adiabatic limit the Friedel phase $\delta_{t}$ and Glazman - Raikh angle $\vartheta_{t}$ are not independent, but connected through (9) and 13

$$
\frac{1}{\delta_{t}} \frac{d \delta_{t}}{d t}=\frac{\pi E_{c}}{4 \Gamma_{0}} \sin 2 \vartheta_{t} \frac{d \vartheta_{t}}{d t}
$$

with $\Gamma_{0}=\pi \rho_{0}\left|v_{0}\right|^{2} \ll E_{c}$. Thus, the Friedel contribution to tunnel current can be expressed in terms of shuttle velocities as follows:

$$
\bar{I}_{0}(t)=\frac{\dot{y}}{\lambda} \frac{e E_{c}}{8 \Gamma_{0}} \cdot \frac{e V_{\text {bias }}}{k_{B} T_{K}(t)} \cdot \frac{\tanh \left(\frac{y-y_{0}}{\lambda}\right)}{\cosh ^{2}\left(\frac{y-y_{0}}{\lambda}\right)}
$$




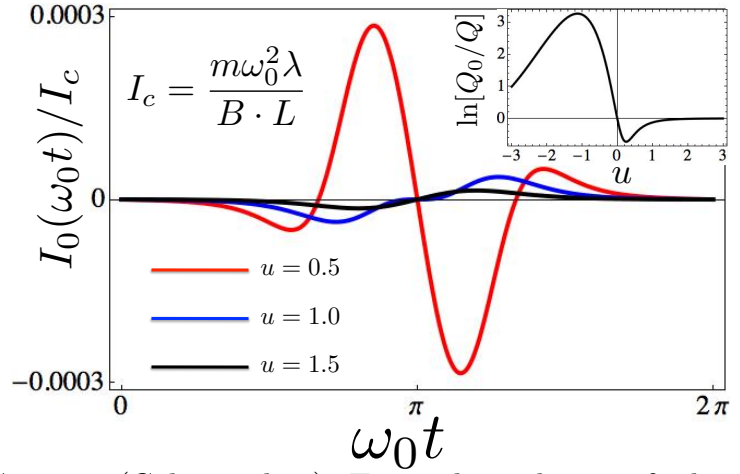

FIG. 2. (Color online) Time dependence of the current $I_{0}$ for different values of asymmetry parameter $u=$ $y_{0} / \lambda$. For all three curves shuttle oscillates with amplitude $y_{\max }=\lambda, \hbar \omega_{0} /\left(k_{B} T_{K}^{\text {min }}\right)=10^{-3},\left|e V_{\text {bias }}\right| /\left(k_{B} T_{K}^{\text {min }}\right)=$ $g \mu_{B} B /\left(k_{B} T_{K}^{m i n}\right)=0.1$ with $T_{K}^{(0))}=2 K, \lambda / L=10^{-4}$. Insert: $\ln \left[Q_{0} / Q\right]$ as a function of $u, Q_{0}=10^{4}$.

Here the time dependence of the tunnel current is predetermined by the time dependence of tunnel integrals for the nanoisland moving in $y$ direction, i.e. by the function $y(t)$ and its derivative $\dot{y}$. Moreover, one can see that even in the case of possible instability large amplitude oscillations are exponentially suppressed. Typical behaviour of $I_{0}(t)$ is shown in Fig.2. The non-sinusoidal form of current is associated with time dependence of both tunnel width and Kondo temperature.

The second term $\bar{I}_{\text {int }}(t)$ given by Eq. 12 leads to "ohmic" contribution to the current with unitary conductance $G_{0}=e^{2} / h$ (see discussion below):

$$
\bar{I}_{\text {int }}(t)=G_{0} V_{\text {bias }} \sin ^{2} 2 \vartheta_{t} \sum \sin ^{2} \delta_{\sigma}
$$

The force in the r.h.s. of the Nevoton equation (2) is a sum of the driving force $F_{0}$, the Laplace force $F_{L}$ and electromotive (emf) force $F_{\text {emf }}$ :

$$
F(y, t)=F_{0}(t)+\overline{\mathcal{I}}_{t} \cdot B \cdot L+F_{\mathrm{emf}}
$$

The emf force can be estimated as $F_{\text {emf }} \sim \dot{y}(B \cdot L)^{2} G_{0}$ 22]. Due to sequential geometry of electric circuit, the current $\overline{\mathcal{I}}_{t}=\bar{I}_{0}(t)+\bar{I}_{\text {int }}(t)$ is the tunneling current defined by (15) and (16). In the limit of small bias voltage $\left|e V_{\text {bias }}\right| \ll k_{B} T_{K}^{m \imath n}$, electrons in the source and the cantilever are supposed to be in adiabatically stationary state of thermal equilibrium. Then the parametrization (15) is valid and with accuracy to small parameters $O\left(\left[\left(e V_{\text {bias }} /\left(k_{B} T_{K}^{m i n}\right)\right]^{2},\left[\left(g \mu_{B} B\right) /\left(k_{B} T_{K}^{m i n}\right)\right]^{2}\right)\right.$ the Lorentz force may be written as

$$
F_{L}=F_{a d}(y(t))-\dot{y} \frac{d F_{a d}}{d y} \frac{\hbar \pi E_{c}}{16 \Gamma_{0} k_{B} T_{K}^{(0)}}
$$

where $F_{a d}=2 B \cdot L \cdot G_{0} \cdot V_{\text {bias }} \cosh ^{-2} \frac{\left[y(t)-y_{0}\right]}{\lambda}$ and $T_{K}^{(0)}$ is a Kondo temperature at equilibrium position. Small correction to the adiabatic Lorentz force in the 18 may be considered as a first term in the expansion over a small non adiabatic parameter $\omega_{0} \tau \ll 1$, where $\tau$ is the retardation time associated with inertia of the Kondo cloud. Using such interpretation one gets $\tau$ :

$$
\tau=\frac{\hbar \pi E_{c}}{16 \Gamma_{0} k_{B} T_{K}^{(0)}}=\frac{1}{2}\left|\frac{Q^{-1}(B)-Q^{-1}(-B)}{\omega(B)-\omega(-B)}\right|
$$

where $Q(B)$ and $\omega(B)$ are the quality factor and oscillator's frequency at finite magnetic field $B$ respectively. Equation Eq 19 allows one to obtain information about dynamics of the Kondo clouds from the analysis of the experimental investigation of the mechanical vibrations. The retardation time associated with dynamics of Kondo cloud is parametrically large compared with the time of formation of the Kondo cloud $\tau_{K}=\hbar / T_{K}$ and can be measured owing to small deviation from adiabaticity. 23. Also we would like to emphasize a supersensitivity of the quality factor to the change of the equilibrium position of the cantilever characterizing by the parameter $y_{0}$. The plot $\ln \left[Q_{0} / Q\right]$ is presented in insert of Fig 2. From this plot one can see that both suppression $Q>Q_{0}$ and enhancement $Q<Q_{0}$ of the dissipation of nanomechanical vibrations (depending on the direction of the magnetic field and the equilibrium position of the cantilever ) can be stimulated by Kondo tunneling. The latter demonstrate potentialities for the Kondo induced electromechanical instability which will be a subject for separate analysis.

Equations (15 18) and (19) represent the central results of the Letter. On the one hand, we have shown that the electric current associated with the Kondo effect results in magnetic field dependent $Q$-factor allowing to finetune the nano-mechanical resonator. On the other hand, the non-ohmic part of the current provides an information about retardation effects related to the motion of the Kondo cloud. Thus, the measurement of the Kondo forces in Single Electron Transistor give a complementary to conductance measurements information.

In conclusion we have shown that the Kondo phenomenon in single electron tunneling gives a very promising and efficient mechanism for electromechanical transduction on a nanometer length scale. Measuring of nanomechanical response on Kondo-transport in nanomechanical single-electronic device enables one to study kinetics of formation of Kondo-screening and offers a new approach for studying nonequilibrium Kondo phenomena. Kondo effect provides a possibility for super high tunability of the mechanical dissipation as well as super sensitive detection of mechanical displacement.

We appreciate illuminating discussions with B.L. Altshuler, P. Brouwer, J. von Delft, Yu. Galperin, S. Ludwig, F. von Oppen and E. Weig. MK acknowledges the hospitality of Institute Henri Poincare (Paris) at the workshop "Disordered Quantum Systems" in April-July 2012, where part of this work has been done. 
[1] A. K. Hüttel, B. Witkamp, M. Leijnse, M. R. Wegewijs, and H. S. J. van der Zant, Phys. Rev. Lett. 102, 225501 (2009)

[2] Y. Azuma, T. Hatanaka, M. Kanehara, T.Teranishi, S. Chorley, J. Prance, C.G. Smith, and Y. Majima, Appl.Phys. Lett. 91, 053120 (2007).

[3] T. Faust, P. Krenn, S. Manus, J.P. Kotthaus, E.M. Weig, Nat. Commun. 3, 728 (2012)

[4] D. Fedorets, L. Y. Gorelik, R. I. Shekhter, M. Jonson, Phys. Rev. Lett. 92, 166801 (2004).

[5] R. I. Shekhter, F. Santandrea, G. Sonne, L. Y. Gorelik, and M. Jonson, Low Temp. Phys. 35, 662 (2009).

[6] R.I. Shekhter, L.Y. Gorelik, L.I. Glazman, and M.Jonson, Phys. Rev. Lett. 97, 156801 (2006)

[7] D. Radic, A. Nordenfelt, A.M.Kadigrobov, R.I.Shekhter, M.Jonson and L.Y.Gorelik, Phys. Rev. Lett. 107, 236802 (2011)

[8] A. Nordenfeld, Y.Tarakanov, L.Y.Gorelik, R.I.Shekhter, and M.Jonson, New J of Phys 12, 123013 (2010)

[9] M.N. Kiselev, K. Kikoin, R.I. Shekhter, and V.M. Vinokur, Phys. Rev. B 74, 233403 (2006)

[10] The form $J_{\alpha \alpha^{\prime}}=4 v_{\alpha}^{*} v_{\alpha^{\prime}} / E_{c}$ of exchange parameter implies a Schieffer-Wolff-like transformation of generic timedependent Anderson Hamiltonian. The adiabatic part of transformation matrix gives this equation. 11] Nonadiabatic corrections $\sim \hbar \omega_{0} / E_{c}, 11,12$ are neglected.

[11] A. Kaminski, Yu.V. Nazarov, and L.I. Glazman, Phys. Rev. B 62, 8154 (2000)

[12] G. Cohen, V. Fleurov, and K. Kikoin, Phys. Rev. B 79, 245307 (2009)

[13] One can notice that the time-dependent Berry-like term $H_{B}$, the first and the second terms of $\delta H$ in $\mathrm{Eq} 4$ form the
$S U(2)$ algebra. The appearance of the Berry phase leads to extra kinematic constraints to be taken into account when the tunnel current is calculated.

[14] T. Aono, Phys. Rev. Lett. 93, 116601 (2004).

[15] M. Pustilnik and L.I. Glazman, J. Phys.: Cond. Mat. 16, R513 (2004).

[16] V. Kashcheyevs, A. Aharony, and O. Entin-Wohlman, Phys. Rev. B 69, 195301 (2004).

[17] D.C. Langreth, Phys. Rev. 150, 516 (1966).

[18] P. Nozières, J. Low Temp. Phys. 17, 31 (1974).

[19] The Nozieres [18] fixed point Fermi Liquid Hamiltonian $H_{\mathrm{Noz}}=\sum_{k \sigma} \tilde{\xi}_{k} \phi_{k \sigma}^{\dagger} \phi_{k \sigma}-\sum_{k k^{\prime} \sigma} \frac{\tilde{\xi}_{k}+\tilde{\xi}_{k}^{\prime}}{2 \pi \rho_{0} k_{B} T_{K}} \phi_{k \sigma}^{\dagger} \phi_{k^{\prime} \sigma}+$ $+\frac{1}{\pi \rho_{0}^{2} k_{B} T_{K}} \sum_{k k^{\prime}} \phi_{k \uparrow}^{\dagger} \phi_{k^{\prime} \uparrow} \sum_{k k^{\prime}} \phi_{k \downarrow}^{\dagger} \phi_{k^{\prime} \downarrow}$ is written in terms of $\phi_{k, \sigma}$ fermions with a bandwidth $k_{B} T_{K}$ and spectrum $\tilde{\xi}_{k}$. It is used for accounting for inelastic contributions to the transport (see 15]).

[20] The Berry-like term cancels out the derivative of $\cos 2 \vartheta_{t}$ in Friedel contribution $\bar{I}_{0}(t)$ and produces an entanglement current arising due to non-commutativity of $\hat{\mathcal{Q}}_{t}$ and $\hat{q}_{t}$. The entanglement current compensate a contribution to $\bar{I}_{\text {int }}(t)$ associated with derivative of $\sin 2 \vartheta_{t}$.

[21] See also [15] for the details of derivation of electric conductance for Kondo problem using Kubo formula.

[22] The smallness of electromotive force is guaranteed by the upper bound on external magnetic field applied to the cantilever $\Phi / \Phi_{0} \cdot L / \lambda<E_{c} / \Gamma_{0} \cdot\left|e V_{\text {bias }}\right| /\left(k_{B} T_{K}\right)$ where $\Phi=B \cdot \mathcal{S}_{\lambda}$ is a flux through the area $\mathcal{S}_{\lambda} \sim \lambda^{2}$ and $\Phi_{0}=h / e$ is a flux quantum. The value of the field corresponding to this bound is roughly estimated as $B_{u} \sim 10 T$.

[23] Parametric largeness of $\tau \gg \tau_{K}$ justifies neglecting corrections of the order of $\omega_{0} \tau_{K} \ll \omega_{0} \tau$ to the scattering phase and the Nozieres Hamiltonian. 